COMMUNICATIONS IN

ANALYSIS AND GEOMETRY

Volume 15, Number 1, 195-216, 2007

\title{
Type II ancient solutions to the Ricci flow on surfaces
}

\author{
Sun-Chin Chu
}

Type II (ancient) solutions to the Ricci flow on surfaces are not yet classified. It is conjectured that the Rosenau solution and the cigar are the only solutions, modulo scaling. In this paper, we mainly study the backward limit and the circumference at spatial infinity of Type II ancient solutions on noncompact surfaces.

\section{Introduction}

Recall that a solution to the Ricci flow is called ancient if it exists on a time interval $(-\infty, \omega)$ containing $t_{0}$ for some $t_{0} \in(-\infty, \infty)$. Let $\left(\mathcal{M}^{n}, g(t)\right)$ be a solution to the Ricci flow. We define what it means to be a Type I or Type II ancient solution as follows:

First, $\left(\mathcal{M}^{n}, g(t)\right)$ is a complete ancient solution with bounded curvature (the bound may depend on time.)

- It is Type I if it satisfies

$$
\sup _{\mathcal{M} \times\left(-\infty, t_{0}\right]}|t||\operatorname{Rm}(x, t)|<\infty .
$$

- It is Type II if it satisfies

$$
\sup _{\mathcal{M} \times\left(-\infty, t_{0}\right]}|t||\operatorname{Rm}(x, t)|=\infty .
$$

Note that hypothesis (1.2) on a Type II ancient solution implies that the metric must be nonflat.

In [11], Hamilton shows that the only Type I ancient solutions on surfaces are the round sphere $\mathbb{S}^{2}$ and the flat plane $\mathbb{R}^{2}$, and their quotients. Therefore, any nonflat complete ancient solution with bounded curvature on a noncompact surface is Type II. That is, there does not exist a Type I nonflat ancient solution on a noncompact surface. As we know so far, Type II ancient solutions on surfaces have not been classified. It is conjectured that 
the noncompact case should correspond to the cigar soliton and compact case to the Rosenau solution [19].

Of particular interest to us is to study Type II ancient solutions on surfaces. By the strong maximum principle, we see that $R(g(t)) \equiv 0$ everywhere and it is flat, or $R(g(t))>0$ everywhere and it is diffeomorphic to $\mathbb{S}^{2}$ or $\mathbb{R}^{2}$. Here and throughout, let $\left(\mathbb{R}^{2}, g(t)\right)$ denote a Type II ancient solution to the Ricci flow. We shall see that such a solution can be extended to a complete eternal solution, i.e., it is defined on $(-\infty, \infty)$. Remark that the curvature is still bounded at each time slice.

The paper is organized as follows. In Section 2, we study the limits backwards in time, in a way analogous to a maximal solution of Type IIb in [11], of Type II ancient solutions on surfaces. Proposition 2.5 shows that the backward limit of such a solution is a multiple of the cigar soliton. In Section 3 , we investigate the asymptotic volume ratio, total curvature, aperture and circumference at spatial infinity of Type II ancient solutions on complete noncompact surfaces. We shall see the scalar curvature of such a solution decays to zero at spatial infinity, hence these quantities are preserved under the Ricci flow. Theorem 3.4 shows that the circumference at spatial infinity of such a solution is finite, therefore, the volume grows linearly. Since Riemann surfaces are Kähler, this improves Ni's theorem [13], namely that any nonflat ancient solution to the Kähler-Ricci flow with bounded nonnegative bisectional curvature has asymptotic volume ratio zero. By the Harnack estimate, the function $R_{\max }(t)=\sup R(\cdot, t)$ is nondecreasing. Does a Type II ancient solution on a surface satisfy $\lim _{t \rightarrow-\infty} R_{\max }(t)>0$ ? Theorem 4.1 gives an affirmative answer to the noncompact case.

\section{Taking limits backwards in time}

In this section, we shall take limits backwards in time of Type II ancient solutions on surfaces.

\subsection{The compactness theorem}

Recall the definition of convergence of pointed solutions to the Ricci flow.

To begin with, we fix a time interval $(\alpha, \omega)$ with $-\infty \leq \alpha<0$ and $0<\omega \leq \infty$

Definition 2.1. A sequence $\left\{\left(\mathcal{M}_{k}^{n}, g_{k}(t), O_{k}\right)\right\}_{k \in \mathbb{N}}, t \in(\alpha, \omega)$, of complete pointed solutions to the Ricci flow converges to a complete pointed solution to the Ricci flow $\left(\mathcal{M}_{\infty}^{n}, g_{\infty}(t), O_{\infty}\right), t \in(\alpha, \omega)$, if there exist 
(1) an exhaustion $\left\{U_{k}\right\}_{k \in \mathbb{N}}$ of $\mathcal{M}_{\infty}$ by open sets with $O_{\infty} \in U_{k}$, and

(2) a sequence of diffeomorphisms $\Phi_{k}: U_{k} \rightarrow V_{k}=\Phi_{k}\left(U_{k}\right) \subset \mathcal{M}_{k}$ with $\Phi_{k}\left(O_{\infty}\right)=O_{k}$ such that $\left(U_{k}, \Phi_{k}^{*}\left[\left.g_{k}(t)\right|_{V_{k}}\right]\right)$ converges in $C^{\infty}$ to $\left(\mathcal{M}_{\infty}, g_{\infty}(t)\right)$ uniformly on compact sets in $\mathcal{M}_{\infty} \times(\alpha, \omega)$.

We review Hamilton's compactness theorem for sequences of solutions to the Ricci flow as follows.

Theorem 2.2. [11]. Let $\left\{\left(\mathcal{M}_{k}^{n}, g_{k}(t), O_{k}\right)\right\}_{k \in \mathbb{N}}, t \in(\alpha, \omega)$, be a sequence of complete pointed solutions to the Ricci flow such that

(i) (uniformly bounded curvatures)

$$
\left|\mathrm{Rm}_{k}\right|_{k} \leq C_{0} \text { on } \mathcal{M}_{k} \times(\alpha, \omega)
$$

for some constant $C_{0}<\infty$ independent of $k$, and

(ii) (injectivity radius estimate at $t=0$ )

$$
\operatorname{inj}\left(O_{k}, g_{k}(0)\right) \geq i_{0}
$$

for some constant $i_{0}>0$.

Then, there exists a subsequence $\left\{j_{k}\right\}_{k \in \mathbb{N}}$ such that $\left\{\left(\mathcal{M}_{j_{k}}, g_{j_{k}}, O_{j_{k}}\right)\right\}$ converges to a complete pointed solution to the Ricci flow $\left(\mathcal{M}_{\infty}^{n}, g_{\infty}(t), O_{\infty}\right)$, $t \in(\alpha, \omega)$, as $k \rightarrow \infty$.

Remark 2.3. (1) In fact, if there is a subsequence $\left(\mathcal{M}_{j_{k}}, g_{j_{k}}(0), O_{j_{k}}\right)$ convergent to a limit $\left(\mathcal{M}_{\infty}, g_{\infty}, O_{\infty}\right)$, then there is a subsequence which converges at all times. (2) For the Ricci flow, it is known that curvature bounds on $(\alpha, \omega)$ imply bounds on all derivatives of the curvature on $[\alpha+\varepsilon, \omega)$ for any $\varepsilon>0$. Thus, we need only to assume the curvature bound for solutions to the Ricci flow.

\subsection{The backward limit}

Let us first take a look at the Rosenau solution [19].

Let $\mathcal{M}^{2}$ be the cylinder $\mathbb{R} \times \mathbb{S}_{1}$, where $\mathbb{S}_{1}$ is the circle of radius 1 . We define a solution $g(x, \theta, t), t<0$, to the Ricci flow on $\mathcal{M}^{2}$ by

$$
g(x, \theta, t)=\frac{\sinh (-t)}{\cosh x+\cosh t}\left(d x^{2}+d \theta^{2}\right) .
$$


It is easy to justify that the solution $g(x, \theta, t)$ extends to a complete ancient solution to the Ricci flow on the sphere $\mathbb{S}^{2}$. This complete ancient solution on $\mathbb{S}^{2}$ is the so-called Rosenau solution.

By straightforward computation, the scalar curvature of the metric is given by

$$
R(x, \theta, t)=\frac{1+\cosh t \cdot \cosh x}{\sinh (-t)(\cosh x+\cosh t)}>0,
$$

and attains its maximum curvature at the poles $x= \pm \infty$ :

$$
R_{\max }(t)=\lim _{|x| \rightarrow \infty} \frac{1+\cosh t \cdot \cosh x}{\sinh (-t)(\cosh x+\cosh t)}=\operatorname{coth}(-t)>0
$$

for all $t<0$. Since $\lim _{t \rightarrow 0^{-}} R_{\max }(t)=\infty$, the Rosenau solution is ancient, but not eternal. Note that the Rosenau solution has a Type I singularity as $t \nearrow 0$. By the fact that

$$
\lim _{t \rightarrow 0^{-}} \frac{R(x, \theta, t)}{R_{\max }(t)}=1 \quad \text { for all }(x, \theta) \in \mathbb{R} \times \mathbb{S}_{1},
$$

the normalized solution converges to the round sphere $\mathbb{S}^{2}$ as $t \rightarrow 0^{-}$. On the other hand, we have

$$
\sup _{\mathbb{S}^{2} \times(-\infty,-1]}|t| R=\sup _{(-\infty,-1]}|t| \operatorname{coth}(-t)=\infty
$$

which means that it is a Type II ancient solution on $\mathbb{S}^{2}$.

To study the limits backwards in time, in a way analogous to a maximal solution of Type IIb in [11], of Type II ancient solutions on surfaces, we need the following.

Lemma 2.4. Suppose that $\left(\mathcal{M}^{n}, g(t)\right)$ is a Type II ancient solution and satisfies the injectivity radius bound, namely

$$
\operatorname{inj}(\mathcal{M}, g(t)) \geq \frac{c}{\sqrt{\mathcal{K}(t)}} \text { at each time } t
$$

where $c$ is a positive constant and

$$
\mathcal{K}(t)=\sup _{x \in \mathcal{M}}|\operatorname{Rm}(x, t)| .
$$

Then, there exists a sequence of dilations of the solution which converges to a Type II singularity model. 
Proof. Let $\left\{\gamma_{j}\right\}$ be any sequence with $\gamma_{j} \nearrow 1$, and choose any sequence of time $T_{j} \searrow-\infty$, and pick $\left(x_{j}, t_{j}\right) \in \mathcal{M} \times\left[T_{i}, 0\right]$ such that

$$
\left|t_{j}\right|\left(t_{j}-T_{j}\right)\left|\operatorname{Rm}\left(x_{j}, t_{j}\right)\right| \geq \gamma_{j} \sup _{M \times\left[T_{j}, 0\right]}|t|\left(t-T_{j}\right)|\operatorname{Rm}(x, t)| .
$$

Now consider the dilated solutions

$$
g_{j}(t)=\left|\operatorname{Rm}\left(x_{j}, t_{j}\right)\right| \cdot g\left(t_{j}+\frac{t}{\left|\operatorname{Rm}\left(x_{j}, t_{j}\right)\right|}\right) .
$$

By the injectivity radius bound (2.1) and definition of $g_{j}$, we obtain

$$
\operatorname{inj}\left(x_{j}, g_{j}(0)\right) \geq c .
$$

Each solution $g_{j}$ exists on the time interval $\left(-\infty, \frac{\omega-t_{j}}{\mid \operatorname{Rm}\left(x_{j}, t_{j}\right)}\right)$, which contains the subinterval $\left[-\alpha_{j}, \omega_{j}\right]$ with

$$
\alpha_{j}=\left(t_{j}-T_{j}\right)\left|\operatorname{Rm}\left(x_{j}, t_{j}\right)\right| \quad \text { and } \quad \omega_{j}=-t_{j}\left|\operatorname{Rm}\left(x_{j}, t_{j}\right)\right| .
$$

By definition, we have

$$
\begin{aligned}
\frac{1}{1 / \alpha_{j}+1 / \omega_{j}}= & \frac{\alpha_{j} \omega_{j}}{\alpha_{j}+\omega_{j}}=\frac{\left|t_{j}\right|\left(t_{j}-T_{j}\right)\left|\operatorname{Rm}\left(x_{j}, t_{j}\right)\right|}{\left|T_{j}\right|} \\
& \geq \gamma_{j} \sup _{M \times\left[T_{j}, 0\right]} \frac{|t|\left(t-T_{j}\right)|\operatorname{Rm}(x, t)|}{\left|T_{j}\right|} \\
& \rightarrow \infty \quad \text { as } j \rightarrow \infty \quad \text { since } T_{j} \rightarrow-\infty .
\end{aligned}
$$

This implies that $\lim _{j \rightarrow \infty} \alpha_{j}=\infty$ and $\lim _{j \rightarrow \infty} \omega_{j}=\infty$. Therefore, for any given $\alpha, \omega>0$, the interval $\left(-\alpha_{j}, \omega_{j}\right)$ contains the subinterval $(-\alpha, \omega)$ for $j$ sufficiently large.

On the other hand, for all $(x, t) \in \mathcal{M} \times(-\alpha, \omega)$, we see that

$$
\begin{aligned}
\left|\operatorname{Rm}_{j}(x, t)\right|= & \frac{1}{\left|\operatorname{Rm}\left(x_{j}, t_{j}\right)\right|}\left|\operatorname{Rm}\left(x, t_{j}+\frac{t}{\left|\operatorname{Rm}\left(x_{j}, t_{j}\right)\right|}\right)\right| \\
& \leq \frac{\left(t_{j}-T_{j}\right)\left|t_{j}\right|}{\gamma_{j}\left(t_{j}-T_{j}+\frac{t}{\left|\operatorname{Rm}\left(x_{j}, t_{j}\right)\right|}\right)\left|t_{j}+\frac{t}{\left|\operatorname{Rm}\left(x_{j}, t_{j}\right)\right|}\right|} \\
& =\frac{\alpha_{j} \omega_{j}}{\gamma_{j}\left(\alpha_{j}+t\right)\left(\omega_{j}-t\right)}
\end{aligned}
$$

uniformly bounded for $j$ sufficiently large since $\alpha_{j} \rightarrow \infty, \omega_{j} \rightarrow \infty$ and $\gamma_{j} \nearrow 1$. 
Consequently, we conclude that the sequence $\left(\mathcal{M}, g_{j}(t), x_{j}\right),-\alpha<t<\omega$, satisfies the hypotheses of Hamilton's compactness theorem. It follows that there exits a subsequence of $\left(\mathcal{M}, g_{j}(t), x_{j}\right)$ which limits to a complete pointed eternal solution $(\overline{\mathcal{M}}, \bar{g}(t), \bar{x})$ satisfying

$$
\sup _{\overline{\mathcal{M}} \times(-\infty, \infty)}|\operatorname{Rm}| \leq 1=|\operatorname{Rm}(\bar{x}, 0)|,
$$

that is, the limit is a Type II singularity model. The lemma follows.

Since a Type II ancient solution on a surface has positive curvature everywhere, the metric satisfies the injectivity radius bound (2.1). Lemma 2.4 implies that the backward limit of such a solution is a Type II singularity model. By construction, the only curvature is positive and attains its maximum in space-time, therefore, it follows from [11] that the limit must be a multiple of the cigar soliton. We conclude this section with the following proposition.

Proposition 2.5. If a complete ancient solution to the Ricci flow on a surface with bounded curvature is not a quotient of the round sphere or of the flat plane, then the ancient solution is Type II. Moreover, the backward limit of such a solution is a multiple of the cigar soliton.

As a corollary, we see that the backward limit of the Rosenau solution is the cigar soliton.

Remark 2.6. Proposition 2.5 is also obtained independently by Chow et al. [4].

\section{The geometry at spatial infinity of Type II ancient solutions on $\mathbb{R}^{2}$}

In this section, first we recall the asymptotic volume ratio, total curvature, aperture and circumference at infinity of complete noncompact surfaces with bounded positive curvature. Next, we study these quantities of Type II ancient solutions on $\mathbb{R}^{2}$. We shall see that these quantities are preserved under the Ricci flow. $\mathrm{Ni}$ [13] proves that any nonflat ancient solution to the Kähler-Ricci flow with bounded nonnegative bisectional curvature has asymptotic volume ratio zero. For Riemann surfaces, Theorem 3.4 improves Ni's theorem since finite circumference at infinity implies that the volume grows linearly, hence that the asymptotic volume ratio is zero. 


\subsection{The geometry of complete surfaces at infinity}

Suppose that $\left(\mathcal{M}^{n}, g\right)$ is a complete Riemannian manifold with nonnegative Ricci tensor. The Bishop-Gromov theorem says that the function

$$
r \rightarrow \frac{\operatorname{Vol}(B(p, r))}{\omega_{n} r^{n}}
$$

where $B(p, r)=\{x \mid \operatorname{dist}(x, p)<r\}$, is monotone decreasing for any $p \in \mathcal{M}$. The asymptotic volume ratio $\alpha_{g}$ is defined by

$$
\alpha_{g}=\lim _{r \rightarrow \infty} \frac{\operatorname{Vol}(B(p, r))}{\omega_{n} r^{n}},
$$

which is independent of $p$ and invariant under dilation. It is known that

$$
\alpha_{g} \omega_{n} r^{n} \leq \operatorname{Vol}(B(p, r)) \leq \omega_{n} r^{n} .
$$

Suppose that $\left(\mathbb{R}^{2}, g\right)$ is a complete surface with bounded positive curvature. Let $o \in \mathbb{R}^{2}$ be some point which we call the origin. Denote by $B_{s}$ the open ball of radius $s$ around the origin $o, \ell(s)$ the length of $\partial B_{s}$ and $A(s)$ the area of $B_{s}$. Recall that the total curvature $\tau_{g}$ and aperture $\mathcal{A}_{g}$ of the metric $g$ are given by

$$
\tau_{g}=\int_{\mathbb{R}^{2}} K d \mu_{g} \quad \text { and } \quad \mathcal{A}_{g}=\lim _{s \rightarrow \infty} \frac{\ell(s)}{s},
$$

respectively. Note that the aperture is also independent of the choice of the origin and invariant under dilation. It follows from the Hartman theorem [12] that we have

$$
\lim _{s \rightarrow \infty} \frac{\ell(s)}{s}=2 \pi-\int_{\mathbb{R}^{2}} K d \mu_{g}
$$

and

$$
\lim _{s \rightarrow \infty} \frac{A(s)}{2 s^{2}}=2 \pi-\int_{\mathbb{R}^{2}} K d \mu_{g}
$$

By the Cohn-Vossen theorem, the right-hand side of (3.2a) is nonnegative for a complete noncompact convex surface, that is, the total curvature is at most $2 \pi$. Clearly, the left-hand side of $(3.2 \mathrm{~b})$ is a multiple of the asymptotic volume ratio. 
From (3.2a) and (3.2b), for complete noncompact convex surfaces we see that the aperture is positive if and only if the asymptotic volume ratio is positive. Since Riemann surfaces are Kähler, for a complete nonflat ancient solution with bounded curvature on $\mathbb{R}^{2}$, combining (3.2a) and (3.2b) to Ni's theorem [13] shows that the aperture is also zero. Therefore, we have that if the aperture of a complete ancient solution with bounded curvature on $\mathbb{R}^{2}$ is positive, then the metric is flat.

Since the scalar curvature of a Type II ancient solution on $\mathbb{R}^{2}$ is positive and bounded, by the Bernstein-Bando-Shi estimates, injectivity radius estimate and the fact that the total curvature is at most $2 \pi$, the scalar curvature of such solutions decays to zero at spatial infinity. It follows from [11] that the aperture and asymptotic volume ratio are preserved under the Ricci flow. Consequently, the total scalar curvature is also preserved under the flow.

Now recall that the circumference at infinity of a complete noncompact surface $\left(\mathcal{M}^{2}, g\right)$ is defined by

$$
\mathcal{C}_{g}=\sup _{K} \inf _{U}\{\ell(\partial U) \mid \forall \text { compact set } K \subset \mathcal{M}, \forall \text { open set } U \supset K\} \text {. }
$$

For any monotone sequence of compact sets $K_{n}$ exhausting a complete noncompact surface, we see that

$$
\mathcal{C}_{g} \geq \sup _{n} \inf _{U \supset K_{n}} \ell(\partial U)
$$

On the other hand, for any compact set $K$, there exists a $K_{n}$ with $K \subset K_{n}$ so that we have

$$
\inf _{U \supset K} \ell(\partial U) \leq \inf _{U \supset K_{n}} \ell(\partial U)
$$

Hence, it follows that

$$
\mathcal{C}_{g}=\sup _{n} \inf _{U \supset K_{n}} \ell(\partial U)=\lim _{n \rightarrow \infty} \inf _{U \supset K_{n}} \ell(\partial U)
$$

Since the scalar curvature of a Type II ancient solution on $\mathbb{R}^{2}$ vanishes at spatial infinity, for any time interval $[a, b]$ containing $t_{0}$ there exists a monotone exhaustion sequence of compact sets $K_{n}$ with

$$
0<R(x, t)<\frac{1}{n} \quad \text { for }(x, t) \in\left(\mathbb{R}^{2} \backslash K_{n}\right) \times[a, b] .
$$


Let $\gamma(s)$ be a fixed parameterized curve on $\mathbb{R}^{2}$. Then the length evolves by the formula

$$
\frac{d}{d t} \ell_{g(t)}(\gamma)=\frac{d}{d t} \int_{\gamma} \sqrt{g\left(\partial_{s}, \partial_{s}\right)} d s=\int_{\gamma}-\frac{R}{2} \sqrt{g\left(\partial_{s}, \partial_{s}\right)} d s .
$$

This implies that

$$
-\frac{1}{2 n} \ell_{g(t)}(\partial U) \leq \frac{d}{d t} \ell_{g(t)}(\partial U)<0
$$

for any set $U$ with $K_{n} \subset U$ and $t \in[a, b]$. Therefore, we have

$$
e^{-(1 / 2 n)(t-a)} \inf _{U \supset K_{n}} \ell_{g(a)}(\partial U) \leq \inf _{U \supset K_{n}} \ell_{t}(\partial U) \leq e^{-(1 / 2 n)(b-t)} \inf _{U \supset K_{n}} \ell_{g(b)}(\partial U)
$$

for $t \in[a, b]$.

We conclude this with the following

Lemma 3.1. If the circumference at infinity of an ancient solution (with bounded curvature at each time slice) on $\mathbb{R}^{2}$ is finite for some $t_{0}$, then it is constant in time.

To explore the aperture and circumference at infinity of Type II ancient solutions on $\mathbb{R}^{2}$, we employ the theory of isometric embedding to the surface $\left(\mathbb{R}^{2}, g(t)\right)$ as follows.

In [18], Pogorelov shows that every complete smooth metric with positive curvature, given on a plane, is realizable as an unbounded smooth convex surface $\mathcal{M}^{2}$ in $\mathbb{R}^{3}$. By a result of Stoker [21], coordinates in $\mathbb{R}^{3}$ can be so chosen that $\{z=0\} \equiv \mathbb{R}^{2}$ is a supporting hyperplane to $\mathcal{M}$ at the origin $O=(0,0,0) \in \mathbb{R}^{3}$, and $\mathcal{M}$ is the graph of a nonnegative strictly convex function $f: \Omega \subset\{z=0\} \rightarrow \mathbb{R}$, where $\Omega$ is the image of $\mathcal{M}$ under the orthogonal projection $\pi: \mathbb{R}^{3} \rightarrow\{z=0\}$. Let $o \in \mathbb{R}^{2}$ denote the preimage of the origin $O$ under the embedding. Thus, we can identify the point $o$ with the origin $O$. In what follows, we shall freely realize without explicit mention a pointed surface $\left(\mathbb{R}^{2}, g, o\right)$ as the graph of a nonnegative strictly convex smooth function $f$ as above.

Now take the graph of $f$ over the sublevel set $\{f \leq n\}$ as the compact set $K_{n}$. By a result of Greene and Shiohama [7], the length of level sets is monotone increasing. Together with (3.3) and the observation that $\inf _{U \supset K_{n}} \ell(\partial U)=\ell(\{f=n\})$, this implies that $\mathcal{C}_{g}=\ell(\partial \Omega)$.

There is an essential difference between surfaces with $\tau=2 \pi$ and surfaces with $\tau<2 \pi$. Considering a convex cone as an example, it is known 
that a complete metric with positive curvature given on a plane may be realized by unbounded convex surface in more than one way. This is always the case [17] if the total curvature of the manifold is less than $2 \pi$. On the other hand, it is known [18] that for any complete noncompact surface of nonnegative curvature with $\tau=2 \pi$, there is a unique complete convex surface in $\mathbb{R}^{3}$ isometric to it up to congruence. In particular, if $\mathcal{C}_{g}<\infty$, say $\mathcal{C}_{g}=2 \pi$, then the embedded surface is inside a circular cylinder of radius $\pi$. This implies that the tangent cone of the surface is a ray, thus we see that $\mathcal{A}_{g}=0$ and $\tau_{g}=2 \pi$ by $(3.2 \mathrm{a})$. Therefore, we have that the embedding is always rigid if we have $\mathcal{C}_{g(t)}<\infty$.

As a corollary of Lemma 3.1, we have the following

Lemma 3.2. The isometric embedding of a Type II ancient solution $\left(\mathbb{R}^{2}, g(t)\right)$ is rigid for all $t$ provided that we have $\mathcal{C}_{g\left(t_{0}\right)}<\infty$ for some $t_{0} \in(-\infty, \omega)$.

Remark 3.3. In [5], Daskalopoulos and Hamilton introduce the width $w(g)$ of a metric $g$ on the plane. Let $F: \mathbb{R}^{2} \rightarrow[0, \infty)$ denote a proper function, i.e., $F^{-1}(c)$ is compact for every $c \in[0, \infty)$. The width of $F$ is given by

$$
w(F)=\sup _{c} \ell(\{F=c\}) .
$$

Then, the width $w(g)$ is given by the infimum of $w(F)$ over all smooth proper functions $F$, that is

$$
w=\inf _{F} w(F)
$$

It is clear that if the metric is complete and has positive curvature, then we have

$$
w(g)=\mathcal{C}_{g}
$$

since the surface is realizable as the graph of a (proper) strictly convex function.

\subsection{Circumference of $\left(\mathbb{R}^{2}, g(t)\right)$ at infinity}

For Riemann surfaces, Theorem 3.4 improves Ni's theorem since the finiteness of circumference at infinity implies that the volume grows linearly. Consequently, we see that the asymptotic volume ratio is 0 .

Theorem 3.4. The circumference at spatial infinity of a Type II ancient solution $\left(\mathbb{R}^{2}, g(t)\right)$ is finite and independent of time. 
Proof. Let us begin the proof with the following observation.

Lemma 3.5. Suppose that $\left(\mathbb{R}^{2}, g_{0}\right)$ is a complete surface with positive and bounded curvature. Then, there exists a positive constant $C$ independent of $x \in \mathbb{R}^{2}$ and $r>0$ such that

$$
\frac{1}{\operatorname{Vol}(B(x, r))} \int_{B(x, r)} R d \mu \leq \frac{C}{r}
$$

Proof. We may assume without loss of generality that $R(x) \leq 2$ for all $x$. By the injectivity radius estimate of Meyer and Gromoll, $\operatorname{inj}\left(\mathbb{R}^{2}, g_{0}\right)$ has a lower bound $\pi$. Therefore, it follows from Yau's theorem that there exists a positive constant $C$ (independent of $x$ ) such that

$$
\operatorname{Vol}(B(x, r)) \geq C r \quad \text { for } r \geq 1 \text {. }
$$

Combining this estimate with the Cohn-Vossen theorem shows that

$$
\frac{1}{\operatorname{Vol}(B(x, r))} \int_{B(x, r)} R d \mu \leq \frac{1}{\operatorname{Vol}(B(x, r))} \int_{\mathbb{R}^{2}} R d \mu \leq \frac{4 \pi}{C r} \quad \text { for } r \geq 1 .
$$

On the other hand, for $r<1$, it is easy to see that

$$
\frac{1}{\operatorname{Vol}(B(x, r))} \int_{B(x, r)} R d \mu \leq \frac{1}{\operatorname{Vol}(B(x, r))} \int_{B(x, r)} 2 d \mu=2 .
$$

Therefore, the lemma follows.

Remark 3.6. Since Riemann surfaces are Kähler, it follows from Shi's theorem [20] that the ancient solution $g(t)$ can be extended to an eternal solution still with bounded curvature at each time slice. This fact plays a role in the proof of Lemma 3.8.

For convenience, let $k(x, r)$ denote the average of the scalar curvature over $B(x, r)$ with respect to $g(0)$, that is,

$$
k(x, r)=\frac{1}{\operatorname{Vol}(B(x, r))} \int_{B(x, r)} R_{g(0)} d \mu_{g(0)} .
$$

From the fact that the aperture of a Type II ancient solution on $\mathbb{R}^{2}$ is zero, we have $\tau=2 \pi$ by $(3.2 \mathrm{~b})$, therefore, the isometric embedding of the surface $\left(\mathbb{R}^{2}, g(t)\right)$ is rigid. Let $o$ and $f$ as given in Section 3.1. Denote the sublevel set $\{f \leq n\}$ by $\Omega$, and let $\rho=\operatorname{dist}(o, \partial \Omega)$ and $r=\max _{p \in \partial \Omega} \operatorname{dist}(o, p)$. It is clear that $\Omega \hookrightarrow\left(\mathbb{R}^{2}, g, o\right)$ is a compact domain with non-empty convex 
boundary $\partial \Omega$. We may combine the results of Lemma 35.3 .1 and Theorem 35.3.2 in [2] to conclude that $\Omega$ has a boundary star-like with respect to $o$ and satisfies

$$
\operatorname{Vol}(\Omega) \geq \frac{\rho}{2} \ell(\partial \Omega)
$$

Combining this estimate with the facts that

$$
\rho \leq r \leq \rho+\ell(\partial \Omega) \quad \text { and } \quad \ell(\partial \Omega)=o(r) \quad \text { as } r \rightarrow \infty
$$

we obtain

$$
\operatorname{Vol}(B(o, r)) \geq \operatorname{Vol}(\Omega) \geq \frac{\rho}{2} \ell(\partial \Omega)>\frac{r}{4} \ell(\partial \Omega)
$$

for $r$ sufficiently large, and hence

$$
r \cdot \frac{1}{\operatorname{Vol}(B(o, r))} \int_{B(o, r)} R d \mu<r \cdot \frac{4}{r \ell(\partial \Omega)} \int_{\mathbb{R}^{2}} R d \mu=\frac{8 \pi}{\ell(\partial \Omega)}
$$

Therefore, we obtain the following.

Lemma 3.7. Let $\Gamma$ be the level curve $\{f=n\}$ and $r=\max _{p \in \Gamma} \operatorname{dist}(p, o)$. Then, for $r$ sufficiently large, we have

$$
r k(o, r)<\frac{8 \pi}{\ell(\Gamma)}
$$

On the basis of an observation of Ni and Tam (Proposition 2 in [16]), we have the following.

\section{Lemma 3.8.}

$$
\limsup _{r \rightarrow \infty} r k(o, r)>0
$$

Proof. The proof proceeds along the same lines as in [16]. For the convenience of the reader, we give the proof here. 
To obtain a contradiction, we suppose that

$$
\limsup _{r \rightarrow \infty} r k(o, r)=0
$$

that is,

$$
k(o, r)=o\left(\frac{1}{r}\right) \quad \text { as } r \rightarrow \infty .
$$

We shall see that this, in conjunction with Lemma 3.5, suffices to claim that the surface must be flat, in contradiction with hypothesis (1.2) on a Type II ancient solution.

Let $\left(\mathcal{M}^{m}, g_{\alpha \bar{\beta}}(x, t)\right)$ be a solution to the Kähler-Ricci flow. Denote by $F(x, t)$ the $\log$ of the volume element, that is,

$$
F(x, t)=\log \left(\frac{\operatorname{det}\left(g_{\alpha \bar{\beta}}(x, t)\right)}{\operatorname{det}\left(g_{\alpha \bar{\beta}}(x, 0)\right)}\right) .
$$

We thus have

$$
F(x, t)=-\int_{0}^{t} R(x, \tau) d \tau .
$$

For convenience, let $\mathfrak{m}(t)=\inf _{\mathcal{M}} F(\cdot, t)$.

Sublemma 3.9 [16]. Suppose $\left(\mathcal{M}^{n}, g_{\alpha \bar{\beta}}\right)$ is a complete noncompact Kähler manifold with nonnegative and bounded bisectional curvature, and the average function $k$ satisfies estimate

$$
k(x, r) \leq \frac{C}{r} .
$$

If there exists some point $x_{0} \in \mathcal{M}$ such that

$$
k\left(x_{0}, r\right)=o\left(\frac{1}{r}\right) \quad \text { as } r \rightarrow \infty
$$

then we have

$$
\lim _{t \rightarrow \infty} \frac{-F(x, t)}{t}=0
$$

and

$$
\lim _{t \rightarrow \infty} R(x, t)=0
$$

for all $x \in \mathcal{M}$. 
Proof. Recall that Shi's theorem [20] implies that the ancient solution $g(t)$ can be extended to an eternal solution. Since $k\left(x_{0}, r\right)=o(1 / r)$ implies $k(x, r)=o(1 / r)$ for all $x \in \mathcal{M}$, it suffices to prove that estimates (3.10) and (3.11) are valid for $x_{0}$.

It follows from Theorem 7.10 in [20], Corollary 2.1 in [14] and estimate (3.8) that we have

$$
-\mathfrak{m}(t) \leq C t^{1 / 2}(1-\mathfrak{m}(t))^{1 / 2}
$$

hence that

$$
1-\mathfrak{m}(t) \leq C(1+t) \quad \text { for all } t .
$$

This, together with Theorem 2.1 in [14], implies that

$$
\begin{aligned}
-F\left(x_{0}, t\right) & \leq C\left[\left(1+\frac{t(1-\mathfrak{m}(t))}{r^{2}}\right) \int_{0}^{r} s k\left(x_{0}, s\right) d s-\frac{t \mathfrak{m}(t)(1-\mathfrak{m}(t))}{r^{2}}\right] \\
& \leq C\left[\left(1+\frac{t^{2}}{r^{2}}\right) \int_{0}^{r} s k\left(x_{0}, s\right) d s+\frac{t^{3}}{r^{2}}\right]
\end{aligned}
$$

for some constant $C$. By (3.9), for any given $\varepsilon>0$, there exists a positive constant $r_{0}$ such that $k\left(x_{0}, r\right) \leq \varepsilon / r$ whenever $r>r_{0}$. Putting $r=t / \sqrt{\varepsilon}$ in the above inequality, we get

$$
\begin{aligned}
-F\left(x_{0}, t\right) & \leq C\left[(1+\varepsilon) \int_{0}^{r_{0}} s k\left(x_{0}, s\right) d s+(1+\varepsilon) \varepsilon\left(r-r_{0}\right)+\varepsilon t\right] \\
& \leq C\left[\int_{0}^{r_{0}} \operatorname{sk}\left(x_{0}, s\right) d s+\sqrt{\varepsilon} t+\varepsilon t\right]
\end{aligned}
$$

for $t$ sufficiently large. By dividing both sides of the inequality by $t$, and letting $t \rightarrow \infty$ and then $\varepsilon \rightarrow 0$, we conclude that estimate (3.10) holds for $x_{0}$.

The trace Harnack inequality says that the function $t \mapsto t R(x, t)$ is increasing in time, hence that

$$
s R(x, s) \cdot \frac{1}{t} \leq R(x, t) \quad \text { for } 0<s<t .
$$

Integrate over $t$ from 1 to $2 s$ to get

$$
s R(x, s) \ln 2 s \leq \int_{1}^{2 s} R(x, t) d t=-F(x, 2 s)-\int_{0}^{1} R(x, t) d t \quad \text { by }(3.7) .
$$


Then using estimate (3.10), we have

$$
R(x, s) \leq \frac{-F(x, 2 s)}{s \ln 2 s}-\frac{\int_{0}^{1} R(x, t) d t}{s \ln 2 s}=o\left(\frac{1}{s}\right) \quad \text { as } s \rightarrow \infty,
$$

and hence estimate (3.11) follows.

Applying the sublemma to the Riemann surface $\left(\mathbb{R}^{2}, g(t)\right)$ gives

$$
\lim _{t \rightarrow \infty} R(x, t)=0 \quad \text { for all } x \in \mathbb{R}^{2},
$$

which, together with the Harnack estimate, shows that $R(x, t) \equiv 0$ everywhere. This leads to a contradiction. The result follows.

By Lemma 3.7, we conclude that

$$
\lim _{r \rightarrow \infty} r k(o, r)=0
$$

provided that the circumference of the solution at infinity is infinite. The theorem follows, since this is a contradiction of the fact that

$$
\limsup _{r \rightarrow \infty} r k(o, r)>0
$$

\section{The lower bound on $R_{\max }$}

Since the scalar curvature of such solutions decays to zero at spatial infinity, the scalar curvature attains its maximum at each time slice. By the Harnack estimate, the function $R_{\max }(t)=\max R(\cdot, t)$ is nondecreasing. Does a Type II ancient solution on a surface satisfy $\lim _{t \rightarrow-\infty} R_{\max }(t)>0$ ? The main result of this section, Theorem 4.1 below, gives an affirmative answer to the noncompact case. By Theorem 3.4, we may assume without loss of generality that $\mathcal{C}=2 \pi$.

Theorem 4.1. If $\left(\mathbb{R}^{2}, g(t)\right)$ is a Type II ancient solution with $\mathcal{C}=2 \pi$, then we have $R_{\max }(t) \geq 4$ for all $t$.

Proof. By the strong maximum principle, and the fact that the surface is nonflat, we have $R(g(t))>0$ for all $t$. Since $\mathcal{A}=0$ and $\mathcal{C}=2 \pi$, it follows 
from Theorem 7.11 in [3] that we have the following splitting:

$$
\left(\mathbb{R}^{2}, g(t), p_{i}\right) \rightarrow \mathbb{R} \times \mathbb{S}^{1} \quad \text { if } p_{i} \rightarrow \infty
$$

which shows that the injectivity radius is at most $\pi$. This implies that the supremum of the sectional curvature on the surface is at least 1 , hence that

$$
2 \leq R_{\max }(g(t))<\infty \text { for all } t
$$

This enables us to take a pointed limit of the sequence $\left(\mathbb{R}^{2}, \tilde{g}_{j}(t), x_{j}\right)$, where the (unnormalized) metric $\tilde{g}_{j}(t)$ is defined by

$$
\tilde{g}_{j}(t)=g\left(t_{j}+t\right)
$$

and points and times $\left(x_{j}, t_{j}\right)$ are chosen as in Lemma 2.4. By Proposition 2.5, there exists a subsequence of $\left(\mathbb{R}^{2}, \tilde{g}_{j}(t), x_{j}\right)$ which limits to a pointed limit $\left(\mathbb{R}^{2}, \tilde{g}(t), O\right)$, which is a multiple of the cigar soliton with

$$
\begin{aligned}
R_{\max }(\tilde{g}) & =R_{\tilde{g}}(O)=\lim _{j \rightarrow \infty} R_{\tilde{g}_{j}}\left(x_{j}\right) \\
& =\lim _{j \rightarrow \infty} R_{g\left(t_{j}\right)}\left(x_{j}\right) \in[2, \infty) .
\end{aligned}
$$

(Note that the limit $\tilde{g}$ is independent of the choice of the sequence $\left(x_{j}, t_{j}\right)$ if it satisfies $R\left(x_{j}, t_{j}\right)=R_{\max }\left(g\left(t_{j}\right)\right)$ and $t_{j} \rightarrow-\infty$.) Therefore, we obtain that

$$
2 \leq R_{\max }(\tilde{g}(t))<\infty .
$$

Indeed, we can improve the lower bound by the following.

Lemma 4.2. The (unnormalized) backward limit $\left(\mathbb{R}^{2}, \tilde{g}(t), O\right)$ is a multiple of the cigar soliton with $\mathcal{C}_{\tilde{g}} \leq 2 \pi$. Moreover, we have $4 \leq R_{\max }(\tilde{g})$.

Combining this estimate with the Harnack estimate, we have

$$
R_{\max }(g(t)) \geq R_{\max }(\tilde{g}) \geq 4
$$

for all $t$ as claimed. Theorem 4.1 follows.

Proof of Lemma 4.2. We first introduce some notation. Let $\bar{g}_{k}$ and $\bar{g}_{k}(t)$ denote the metric $g(-k)$ and the solution $g(t-k)$, respectively. We realize the surface $\left(\mathbb{R}^{2}, \bar{g}_{k}\right)$ as the graph of a nonnegative strictly convex smooth function $f_{k}$ as in Section 3.1. Let $\mathcal{I}_{k}, \mathcal{M}_{k}$ and $p_{k}$ denote the isometric 
embedding, embedded hypersurface and point $\mathcal{I}_{k}^{-1}((0,0,0)) \in \mathbb{R}^{2}$, respectively. Since the total curvature equals $2 \pi$, the embedding $\mathcal{I}_{k}$ is unique up to isometry. By convenient abuse of notation, denote by $\{f \leq n\}$ and $\{f=n\}$ the graphs of any given function $f$ over the sets $\{f \leq n\}$ and $\{f=n\}$, and still call them the sublevel set $\{f \leq n\}$ and level set $\{f=n\}$, respectively.

To show that $\mathcal{C}_{\tilde{g}} \leq 2 \pi$, we investigate the pointed limit of the sequence $\left(\mathbb{R}^{2}, \bar{g}_{k}(t), p_{k}\right)$ as follows.

Sublemma 4.3. The sequence $\left(\mathbb{R}^{2}, \bar{g}_{k}(t), p_{k}\right)$ converges to a complete pointed limit $\left(\mathbb{R}^{2}, \bar{g}(t), p\right)$ with $\mathcal{C}_{\bar{g}} \leq 2 \pi$. Consequently, we have $\mathcal{A}_{\bar{g}}=0$ and $\tau_{\bar{g}}=$ $2 \pi$. Furthermore, the embedding $\mathcal{I}:\left(\mathbb{R}^{2}, \bar{g}(0)\right) \hookrightarrow \mathbb{R}^{3}$ is unique up to congruence.

Proof. Since $\left(\mathbb{R}^{2}, g(t)\right)$ is a Type II ancient solution, the complete pointed surfaces $\left(\mathbb{R}^{2}, \bar{g}_{k}(t), p_{k}\right)$ have uniformly bounded curvature on any given finite interval containing $t=0$ and satisfy the injectivity radius estimate at $t=0$. By Hamilton's compactness theorem, the sequence $\left(\mathbb{R}^{2}, \bar{g}_{k}(t), p_{k}\right)$ subconverges to an eternal solution $\left(\mathbb{R}^{2}, \bar{g}(t), p\right)$ with uniformly bounded curvature. Denote by $\bar{g}$ the metric $\bar{g}(0)$. As noted, the surface $\left(\mathbb{R}^{2}, \bar{g}\right)$ is realizable as the graph of a nonnegative strictly convex smooth function $f$. Let $\mathcal{I}$ and $\mathcal{M}$ denote the isometric embedding and embedded hypersurface, respectively. It is clear that we have $\mathcal{I}(p)=(0,0,0) \in \mathcal{M} \subset \mathbb{R}^{3}$.

By the strong maximum principle we have either $R_{\bar{g}(t)} \equiv 0$ or $R_{\bar{g}(t)}>0$ everywhere in space-time. We now claim that the curvature is positive. The sequence $\left(\mathcal{M}_{k}, \bar{g}_{k}, O\right)$ subconverges to the corresponding embedded pointed surface $(\mathcal{M}, \bar{g}, O)$. Note that if $R_{\bar{g}} \equiv 0$, then the embedded surface must be a cylinder or a plane, which is impossible because the surface $\mathcal{M}_{k}$ is always inside the set $\left\{(x, y, z) \in \mathbb{R}^{3} \mid x^{2}+y^{2} \leq \pi^{2}, z \geq 0\right\}$. Therefore, $R_{\bar{g}}>0$. In other words, the solution $\left(\mathbb{R}^{2}, \bar{g}\right)$ is nonflat as claimed.

Since the graph of $f_{k}$ subconverges to the graph of $f$, the level curve $f_{k}^{-1}\{c\}$ uniformly subconverges to the level curve $f^{-1}\{c\}$. As seen in section 3.1, the length of level curves of $f$ converges to $\mathcal{C}_{\bar{g}}$, thus for any $\eta>0$ there exists a positive constant $c_{0}$ such that

$$
\ell(\{f=c\})>\mathcal{C}_{\bar{g}}-\eta
$$

for all $c \geq c_{0}$. On the other hand, by Theorem 1 in $[1$, p. 225], we have

$$
\liminf _{k \rightarrow \infty} \ell\left(\left\{f_{k}=c\right\}\right) \geq \ell(\{f=c\})
$$


thus there also exits a positive integer $k_{0}$ such that

$$
\ell\left(\left\{f_{k_{0}}=c\right\}\right)>\mathcal{C}_{\bar{g}}-2 \eta
$$

for all $c>c_{0}$. This implies that

$$
\begin{aligned}
2 \pi=\mathcal{C}_{\bar{g}_{k_{0}}}>\ell\left(\left\{f_{k_{0}}=c\right\}\right) \\
>\mathcal{C}_{\bar{g}}-2 \eta
\end{aligned}
$$

for all $\eta>0$. Therefore, we see that $\mathcal{C}_{\bar{g}} \leq 2 \pi$ as claimed. The rest of the proof is immediate from Lemma 3.2 .

Intuitively, the point $p_{k}$ should be close to the point, denoted by $q_{k}$, where the curvature $R_{\bar{g}_{k}}$ attains its maximum. This motivates us to take a pointed limit of the sequence $\left(\mathbb{R}^{2}, \bar{g}_{k}, q_{k}\right)$ and have the following.

Sublemma 4.4. The sequence $\left(\mathbb{R}^{2}, \bar{g}_{k}, q_{k}\right)$ converges to a pointed limit $\left(\mathbb{R}^{2}, \bar{g}, \bar{q}\right)$.

Proof. We first claim that the set $\left\{p_{k}, p_{j}, q_{j}\right\}_{j=k}^{\infty}$ is uniformly bounded in the pointed surface $\left(\mathbb{R}^{2}, \bar{g}_{k}, p_{k}\right), k \geq N$, for some positive integer $N$.

It follows from Sublemma 4.3 that for any given $\varepsilon>0$, there exist a positive number $d>0$ and a positive integer $N$ such that

$$
\int_{\{f<d\}} K_{\bar{g}} d v_{\bar{g}}>2 \pi-\varepsilon
$$

and

$$
\int_{\left\{f_{k}<d\right\}} K_{\bar{g}_{k}} d v_{\bar{g}_{k}}>2 \pi-2 \varepsilon
$$

for $k \geq N$.

Since $R_{\bar{g}_{k}}\left(p_{k}\right) \rightarrow R_{\bar{g}}(O)>0$ as $k \rightarrow \infty$, there exists a positive integer $k_{1} \geq N$ such that

$$
R_{\bar{g}_{k}}\left(p_{k}\right)>\frac{1}{2} R_{\bar{g}}(O)
$$

for $k \geq k_{1}$. Combining (4.3), (4.1) and the Harnack estimate [9], we get the following estimates:

$$
R_{\bar{g}_{k}}\left(q_{j}\right) \geq R_{\bar{g}_{j}}\left(q_{j}\right)=R_{\max }\left(\bar{g}_{j}\right) \geq 2
$$


and

$$
R_{\bar{g}_{k}}\left(p_{j}\right) \geq R_{\bar{g}_{j}}\left(p_{j}\right)>\frac{1}{2} R_{\bar{g}}(O)
$$

for all $j \geq k \geq k_{1}$. As a consequence of the injectivity radius control on $\mathcal{M}_{k}$, gradient estimate on scalar curvature $R_{\bar{g}_{k}}$ and (4.2a), (4.2b), (4.4a), (4.4b), we obtain uniform estimates on

$$
\operatorname{dist}\left(p_{j},\left\{f_{k}<d\right\}\right) \text { and } \operatorname{dist}\left(q_{j},\left\{f_{k}<d\right\}\right)
$$

for all $j \geq k \geq k_{1}$, which implies that the set $\left\{p_{k}, p_{j}, q_{j}\right\}_{j=k}^{\infty}$ is uniformly bounded in the pointed surface $\left(\mathbb{R}^{2}, \bar{g}_{k}, p_{k}\right), k \geq k_{1}$. Therefore, the sequence $\left(\mathbb{R}^{2}, \bar{g}_{k}, q_{k}\right)$ converges to a pointed limit $\left(\mathbb{R}^{2}, \bar{g}, \bar{q}\right)$.

Note that, as pointed out, the sequence $\left(\mathbb{R}^{2}, \bar{g}_{k}, q_{k}\right)$ converges to the pointed limit $\left(\mathbb{R}^{2}, \tilde{g}, p\right)$. By the rotational symmetry of the cigar soliton, we have $\bar{q}=p$ and hence $\tilde{g}$ coincides with $\bar{g}$. From Sublemma 4.3, the circumference of $\left(\mathbb{R}^{2}, \tilde{g}(t)\right)$ at infinity is at most $2 \pi$ as claimed. Since $\left(\mathbb{R}^{2}, \tilde{g}\right)$ is a multiple of the cigar soliton with $\mathcal{C}_{\tilde{g}} \leq 2 \pi$, Lemma 4.2 follows immediately.

As a result of Theorem 4.1, the scalar curvature assumes its maximum in space-time provided that $\mathcal{C}=2 \pi$ and $R(x, t) \leq 4$. This means that the solution is indeed a Type II singularity model. By Hamilton's theorem [10], such a solution must be the cigar soliton. Therefore, we obtain the following.

Corollary 4.5. If $\left(\mathbb{R}^{2}, g(t)\right)$ is a Type II ancient solution with $\mathcal{C}=2 \pi$ and $R(x, t) \leq 4$ for all $(x, t) \in(-\infty, \infty) \times \mathbb{R}^{2}$, then it is the cigar soliton.

We end this section by the following.

Remark 4.6. S. Angenent (see Appendix A in [22]) and L. Wu [22] observe that the logarithmic fast diffusion equation

$$
\frac{\partial}{\partial t} u=\triangle \log u
$$

on the plane $\mathbb{R}^{2}$, where $\triangle$ denotes the Euclidean Laplace operator on $\mathbb{R}^{2}$, represents the evolution of the conformally flat metric with

$$
g=u\left(d x^{2}+d y^{2}\right)
$$

under the Ricci flow. The equivalence follows from the facts that the conformal metric $g$ has scalar curvature $R=-(\triangle \log u) / u$ and in two dimensions $R_{i j}=(1 / 2) R g_{i j}$. Daskalopoulos and Sesum [6] study the classification of eternal solutions of equation (4.5). They show that any positive smooth 
eternal solution $u(x, y, t)$ is a gradient soliton of the form

$$
u(x, y, t)=\frac{2}{\beta\left(\left|x-x_{0}\right|^{2}+\left|y-y_{0}\right|^{2}+\delta e^{2 \beta t}\right)}
$$

for some $\left(x_{0}, y_{0}\right) \in \mathbb{R}^{2}$ and some positive constants $\beta, \delta$, provided that the solution $u$ defines a complete metric of bounded curvature and bounded width. Note that Theorem 3.4 removes the hypothesis of the width being finite.

\section{Acknowledgments}

The author would like to thank Professor Ben Chow for bringing the idea of taking limits backwards in time, Ni's work [13] and Ni and Tam's notes [15, 16] to his attention. The author would also like to thank Professor L. Ni and Professor L.F. Tam for their indispensable notes, and thank Professor Peng Lu for his helpful suggestions and comments. Finally, the author thanks the referee for the helpful suggestions concerning the presentation of this paper.

\section{References}

[1] A. D. Alexandrov and V. A. Zalgaller, Intrinsic geometry of surfaces, Translations of Mathematical Monographs, Vol. 15, AMS, Providence, RI, 1967.

[2] Y. D. Burago and V. A. Zalgaller, Geometric inequality, Springer Series in Soviet Mathematics, Springer-Verlag, Berlin, 1988.

[3] J. Cheeger and T. Colding, Lower bounds on Ricci curvature and the almost rigidity of warped products, Ann. Math. 144 (1996), 189-237.

[4] B. Chow, P. Lu and L. Ni, Hamilton's Ricci flow, Graduate Studies in Mathematics, Vol. 77, AMS, Providence, RI, 2006.

[5] P. Daskalopoulo and R. Hamilton, Geometric estimates for the logarithmic fast diffusion equation, Commun. Anal. Geom. 12 (2004), 143-164.

[6] P. Daskalopoulos and N. Sesum, Eternal solutions to the Ricci flow on $\mathbb{R}^{2}$, Int. Math. Res. Not. 2006, Art. ID 83610, 20 pp.

[7] R. Greene and K. Shiohama, Convex functions on complete noncompact manifolds: topological structure, Invent. Math. 63 (1981), 129-157.

[8] R. Hamilton, The Ricci flow on surfaces, Contem. Math. 71 (1988), $237-261$. 
[9] R. Hamilton, The Harnack estimate for the Ricci flow, J. Diff. Geom. 37 (1993), 225-243.

[10] R. Hamilton, Eternal solutions to the Ricci flow, J. Diff. Geom. 38 (1993), 1-11.

[11] R. Hamilton, The formation of singularities in the Ricci flow, Surveys in Differential Geometry, Vol. 2, International Press, 1995. pp. 7-136.

[12] P. Hartman, Geodesic parallel coordinates in the large, Amer. J. Math. 86 (1964), 705-727.

[13] L. Ni, Ancient solutions to Kähler-Ricci flow, Math. Res. Lett. 12 (2005), 633-653.

[14] L. Ni and L. F. Tam, Kähler-Ricci flow and the Poincaré-Lelong equation, Commun. Anal. Geom. 12 (2004), 111-141.

[15] L. Ni and L. F. Tam, Notes on soliton 1, unpulished.

[16] L. Ni and L. F. Tam, Notes on soliton 2, unpulished.

[17] S. P. Olovjanišnikov, On the bending of infinite convex surfaces, Mat. Sb. 18 (1946), no. 60, 429-440.

[18] A. V. Pogorelov, Extrinsic geometry of convex surface, Translations of Mathematical Monographs, Vol. 35, AMS, Providence, RI, 1973.

[19] P. Rosenau, On fast and super-fast diffusion, Phys. Rev. Lett. 74 (1995), 1056-1059.

[20] W.-X. Shi, Ricci flow and the uniformization on complete noncompact Kähler manifolds, J. Diff. Geom. 45 (1997), 94-220.

[21] J. J. Stoker, Über die Gestalt der positiv gekrümmten offenen Flächen im dreidimensionalen Raum, Comput. Math. 3 (1936), 55-88.

[22] L. F. Wu, The Ricci flow on complete $\mathbb{R}^{2}$, Commun. Anal. Geom. 1, (1993), 439-472.

Department of Mathematics

National Chung Cheng University

CHIA-YI

TAIWAN

E-mail address: scchu@math.ccu.edu.tw

ReCEIVEd April 5, 2006 
